\title{
EFFICIENCY ANALYSIS OF CONTROL ALGORITHMS IN SPATIALLY DISTRIBUTED SYSTEMS WITH CHAOTIC BEHAVIOR
}

\author{
ŁUKASZ KORUS \\ Institute of Computer Engineering, Control and Robotics \\ Wrocław University of Technology, Janiszewskiego 11/17, 50-372 Wrocław, Poland \\ e-mail: lukasz .korus@pwr.wroc.pl
}

\begin{abstract}
The paper presents results of examination of control algorithms for the purpose of controlling chaos in spatially distributed systems like the coupled map lattice (CML). The mathematical definition of the CML, stability analysis as well as some basic results of numerical simulation exposing complex, spatiotemporal and chaotic behavior of the CML were already presented in another paper. The main purpose of this article is to compare the efficiency of controlling chaos by simple classical algorithms in spatially distributed systems like CMLs. This comparison is made based on qualitative and quantitative evaluation methods proposed in the previous paper such as the indirect Lyapunov method, Lyapunov exponents and the net direction phase indicator. As a summary of this paper, some conclusions which can be useful for creating a more efficient algorithm of controlling chaos in spatially distributed systems are made.
\end{abstract}

Keywords: controlling spatiotemporal chaos, coupled map lattice, system stability, Lyapunov exponents, net direction phase.

\section{Introduction}

Deterministic chaos defined as a complex, disordered behavior generated by nonlinear systems (Ott, 2002) is a widely studied phenomenon which has been discovered and observed in practice in many different areas like liquids (Procaccia and Meron, 1986; Langenberg et al., 2004), lasers (Yamada and Graham, 1980; Used and Martin, 2010), plasma (Held et al., 1984; Banerjee et al., 2010), mechanics (Chui and Ma, 1982; Ott, 2002), electronics (Gunaratne et al., 1989; Yim et al., 2004), chemistry (Argoul et al., 1987; Cordoba et al., 2006), biology - the action of the human heart (Govindan et al., 1998) and the brain (Andrzejak et al., 2001; Gautama et al., 2003), and in many others.

There are various approaches to analyze chaotic systems from both theoretical and experimental perspectives. One of them is based on the Takens embedding theory (Takens, 1981) and it is frequently used to analyze chaotic times series (Sauer et al., 1991; Stark, 1999). An extensive review of methods used to analyze experimental, chaotic data can be found in the work of Abarbanel (1996). Another approach based on ergodic theory was studied by Mitkowski and Mitkowski (2012). Many useful indicators, methods and tools can be also found in the work of Lasota and Mackey (1997).

The state trajectory of a chaotic system cannot be predicted due to its sensitivity to initial conditions but, as has been proved, it can be controlled in an efficient way (Ott et al., 1990; Singer et al., 1991; Dressler and Nitsche, 1992; Chen and Dong, 1993; Alsing et al., 1994; Pyragas, 2001; Andrievskii and Fradkov, 2003; Chen et al., 2013; Bashkirtseva and Ryashko, 2013). The term "control of chaos" in this case is considered to be a process of changing irregular and unpredictable behavior to a well-ordered and periodic one. This issue becomes even more essential if we take into consideration that many real systems are in fact spatially distributed. That means that chaotic behavior can be observed both in time and space directions. This phenomenon can be also observed in simple systems like cellular neural networks (CNNs) (Ogorzałek et al., 1996a), large arrays of electronics circuits (Ogorzałek et al., 1996b) or the coupled map lattice (CML), examined in this paper. Controlling chaos in a such type of systems is more complicated because it requires consideration of the additional relation between many components in time and space. Moreover, sensitivity to initial conditions both in time and space may cause unpredictable negative results which, in some 
cases (i.e., chemical reactors), can be adverse or even dangerous.

The main purpose of this paper is to provide comparison results for different, classical control algorithms used to control chaos in spatially distributed systems like the CML. To this end, the entire framework defined in the previous article (Korus, 2011) is used. The comparison is made based on qualitative and quantitative evaluation methods. In the pre-study phase, results of numerical simulation are presented to preliminarily assess if the examined algorithm is able to change behavior generated by the system. Moreover, two coefficients (the Lyapunov exponent and the net direction phase) are introduced to prove the change in the type of behavior generated by the system under control by the examined algorithm. Furthermore, stability analysis for each algorithm is performed to confirm its effectiveness in the area of controlling the chaotic behavior in a spatiotemporal system. To summarize the obtained results, some conclusions regarding the desired properties of an ideal algorithm are provided.

\section{CML: The mathematical model}

The coupled map lattice is a simple mathematical model of a spatially distributed system defined by the following equation (Crutchfield and Kaneko, 1987; Kaneko, 1990):

$$
\begin{aligned}
x_{t+1}(n)= & (1-\varepsilon) g\left(x_{t}(n), p\right) \\
& +\frac{\varepsilon}{2}\left[g\left(x_{t}(n-1), p\right)+g\left(x_{t}(n+1), p\right)\right],
\end{aligned}
$$

where $x_{t+1}(n)$ describes the evolution of components $n=1, \ldots, N$ in discrete time steps $t=1, \ldots, T$, and $\varepsilon$ is a coupling parameter. The coupling function is the logistic equation given by

$$
g(x, p)=p x(1-x),
$$

where $p$ is the bifurcation parameter. System states in particular time step can be written by using the vector

$$
\mathbf{x}_{t}=\left(x_{t}(1), x_{t}(2), \ldots, x_{t}(N)\right) \text {. }
$$

State values for all components at the beginning of simulation, i.e., for $t=1$, are generated from a uniform distribution. In this paper, a CML model with fixed boundary conditions is examined, i.e.,

$$
\begin{aligned}
x(N+1) & =0, \\
x(0) & =0 .
\end{aligned}
$$

As was shown by Korus (2011), for some range of coupling $(\varepsilon)$ and bifurcation $(p)$ parameters, the CML defined by (1)-(4) generates a spatiotemporal chaotic behavior.

\section{Control of chaos}

The aim of the control task in this paper is to change the type of the system's state trajectory from a chaotic to a periodic one. This goal can be achieved by introducing small perturbation to the coupling parameters $\varepsilon=(\varepsilon(1), \varepsilon(2), \ldots, \varepsilon(N))$ or vector of bifurcation parameters $\mathbf{p}=(p(1), p(2), \ldots, p(N))$. Another way to introduce periodic behavior in the system is to add a feedback loop to the evolution function (1). This article tackles the issue of chaos control by an additional feedback function.

The control task can be defined in a more general form (Andrievskii and Fradkov (2003)). Let us consider a system defined by the equations

$$
\begin{aligned}
\mathbf{x}_{t+1} & =F\left(\mathbf{x}_{t}\right)+\mathbf{u}_{t}, \\
\mathbf{y}_{t} & =h\left(\mathbf{x}_{t}\right),
\end{aligned}
$$

where $\mathbf{x}_{t} \in \mathbb{R}^{N}$ is the $N$-dimensional state vector, $\mathbf{u}_{t} \in \mathbb{R}^{M}$ is $M$-dimensional control vector and $\mathbf{y}_{t} \in \mathbb{R}^{L}$ is an $L$-dimensional output vector given for a time step $t$. The dynamics of the system (5) are defined by the evolution function $F$. The output vector $\mathbf{y}_{t}$ is considered to be a set of measurements of the system. In some cases the output vector corresponds directly to state vector $\mathbf{y}_{t} \equiv \mathbf{x}_{t}$. Equations (5) can be used to describe both the types of control in the system: by using the additional feedback loop and parametric control involving a small perturbation of control parameters $\mathbf{u}_{t}=\mathbf{p}_{0}+\delta \mathbf{p}$ or $\mathbf{u}_{t}=\varepsilon_{0}+\delta \varepsilon$.

For a system defined by (5), the issues of chaos control can be defined in mathematical terms. The control of chaos is understood as stabilization of unstable a periodic orbits existing in a state space. Let us assume that periodic trajectory $\tilde{\mathbf{x}}_{t}$ exists in a state space of the system without control $\left(\mathbf{u}_{t} \equiv 0\right)$. This trajectory starts from the point $\tilde{\mathbf{x}}_{0}$ and has a period $T$, which can be written as $\tilde{\mathbf{x}}_{t+T}=\tilde{\mathbf{x}}_{t}$ for each $t \geq 0$. The issue of stabilization of an unstable periodic trajectory is about changing the state of the system from $\mathbf{x}_{t}$ to $\tilde{\mathbf{x}}_{t}$,

$$
\lim _{t \rightarrow \infty}\left(\mathbf{x}_{t}-\tilde{\mathbf{x}}_{t}\right)=0
$$

or the output trajectory from $\mathbf{y}_{t}$ to $\tilde{\mathbf{y}}_{t}$,

$$
\lim _{t \rightarrow \infty}\left(\mathbf{y}_{t}-\tilde{\mathbf{y}}_{t}\right)=0
$$

for each $\mathrm{x}_{t}$ in the system which is the result of iterating from the starting point $\mathbf{x}_{0}$. So, the solution of this issue is to find a proper control term $\mathbf{u}_{t}$ which will fulfill Eqns. (6) or (7) in the shortest period of time.

In general, there are three types of feedback control:

- control with open loop given by

$$
\mathbf{u}_{t}=U\left(\mathbf{x}_{0}, t\right)
$$


- control with state feedback described as

$$
\mathbf{u}_{t}=U\left(\mathbf{x}_{t}\right)
$$

- control with output feedback which can be written as

$$
\mathbf{u}_{t}=U\left(\mathbf{y}_{t}\right)
$$

In this paper, the second type of control is examined. For this type, the following examples of control actions can be applied (Parmananda, 1997; Boukabou and Mansouri, 2005):

$$
\begin{aligned}
& \mathbf{u}_{t}=K\left(\mathbf{x}_{t}-\mathbf{x}_{t-1}\right) \\
& \mathbf{u}_{t}=K\left(\mathbf{x}_{t}-\mathbf{x}_{F}\right) \\
& \mathbf{u}_{t}=K\left(\mathbf{x}_{t}-\mathbf{x}_{t-k}\right),
\end{aligned}
$$

where $K$ is the gain parameter of the control module.

For both the types of control (parametric and with feedback loop), a control algorithm which fulfill Eqns. (6) or (7) can be constructed based on the fact that dynamics of each smooth, nonlinear dynamic system can be approximated in a small neighborhood of a fixed point. According to this principle, a linear approximation within a small neighborhood of the fixed point $x_{F}$ can be constructed:

$$
\left(\mathbf{x}_{t+1}-\mathbf{x}_{F}\right)=J\left(\mathbf{x}_{t}-\mathbf{x}_{F}\right),
$$

where $J \in \mathbb{R}^{N \times N}$ is a Jacobi matrix for the function $F\left(\mathbf{x}_{t}\right)$ of the fixed point $\mathbf{x}_{F}$,

$$
J=\left.\frac{\partial F\left(x_{t}\right)}{\partial x_{t}}\right|_{x_{t}=x_{F}} .
$$

Equations (14) can be also written as

$$
\delta \mathbf{x}_{t+1}=J \delta \mathbf{x}_{t}
$$

where

$$
\delta \mathbf{x}_{t}=\mathbf{x}_{t}-\mathbf{x}_{F} .
$$

By introducing the control term, Eqn. (16) can be written as

$$
\delta \mathbf{x}_{t+1}=J \delta \mathbf{x}_{t}+B \mathbf{u}_{t} .
$$

Let us assume the control with linear feedback defined by

$$
\mathbf{u}_{t}=K \delta \mathbf{x}_{t}=K\left(\mathbf{x}_{t}-\mathbf{x}_{F}\right)
$$

Finally, we have

$$
\begin{gathered}
\delta \mathbf{x}_{t+1}=(J+B K) \delta \mathbf{x}_{t}, \\
\mathbf{x}_{t+1}= \begin{cases}F\left(\mathbf{x}_{t}\right)+\mathbf{u}_{t} & \text { for }\left|K \delta \mathbf{x}_{t}\right|<\varepsilon, \\
F\left(\mathbf{x}_{t}\right) & \text { for }\left|K \delta \mathbf{x}_{t}\right|>\varepsilon .\end{cases}
\end{gathered}
$$

To fulfill the condition given by (6), there is a need to select a vector $K$ which will provide the stability of the matrix $J^{\prime}=(J+B K)$. The method described above is well known in control theory as the pole placement control design method. Even though this issue is common in control theory, due to the instability of periodic orbits in chaotic systems its usage is more complex. An additional difficulty is related to the spatiotemporal nature of chaotic behaviors in the CML. It requires consideration of additional dependencies in time and space, which is not present in classical algorithms.

\section{Detection of chaos}

Two coefficients can be used to provide quantitative evaluation of chaotic properties.

4.1. Lyapunov exponents. Lyapunov exponents provide quantitative information about the separation rate of preliminarily close trajectories (Ott, 2002). For one-dimensional discrete equations given by the formula $x_{t+1}=g\left(x_{t}\right)$, we have

$$
h=\lim _{T \rightarrow \infty} \frac{1}{T} \sum_{t=1}^{T} \ln \left|g^{\prime}\left(x_{t}\right)\right| .
$$

A positive Lyapunov exponent means that chaos is present.

As was calculated by Korus (2011), for the CML defined by (1), Lyapunov exponents are given by

$$
\begin{gathered}
h_{1}(n)=\frac{1}{T} \sum_{t=1}^{T} \ln \mid g^{\prime}\left(x_{t}(n) \mid,\right. \\
h_{2}(n)=\ln (|1-2 \varepsilon|)+\frac{1}{T} \sum_{t=1}^{T} \ln \mid g^{\prime}\left(x_{t}(n) \mid .\right.
\end{gathered}
$$

If the greater of these values $h_{\max }=\max \left(h_{1}, h_{2}\right)$ is positive, then the state trajectory for the component denoted by $n$ is chaotic.

4.2. Net direction phase. The net direction phase introduced by Wei et al. (2000) is an indicator similar to magnetization, which can be defined as

$$
M=\frac{1}{T} \sum_{t=1}^{T} S(t),
$$

where $S(t)=1$ for $x_{t+1}-x_{t} \geq 1$ and $S(t)=-1$ for $x_{t+1}-x_{t}<1$, and $T$ is the iteration number. The direction $S(t)=1$ can be denoted by $S_{\uparrow}$ and $S(t)=-1$ as $S_{\downarrow}$. Whenever $M=0$, the situation can be described as the zero net direction phase. This means that the system trajectory $x_{t}$ switches symmetrically from the up direction phase $\left(S_{\uparrow}\right)$ to the down direction phase $\left(S_{\downarrow}\right)$, 
and the system behaves regularly. If $M \neq 0$, this means that the arrangement of the up and down direction phases is disordered and the system generates non-periodical trajectories.

\section{Stability analysis}

Stability analysis in this paper is performed according to the indirect Lyapunov method for non-linear dynamic systems. It is called indirect because conclusions on the stability of a non-linear dynamic system are formulated based on results of stability analysis obtained for its linear approximation. Let us assume there is a discrete dynamic system

$$
\mathbf{x}_{t+1}=F\left(\mathbf{x}_{t}\right),
$$

where $\mathbf{x}_{t}$ and $\mathbf{x}_{t+1}$ are the state vectors. The first step of the method is to create a linear approximation of the original non-linear system,

$$
\mathbf{x}_{t+1}=J \mathbf{x}_{t}+r,
$$

where $J=\partial F_{i} / \partial x_{j}$ is a Jacobi matrix and $r$ is the infinitesimal remainder which fulfills

$$
\lim _{\mathbf{x}_{t} \rightarrow 0} \frac{r\left(\mathbf{x}_{t}\right)}{\left\|\mathbf{x}_{t}\right\|}=0 \text {. }
$$

Thus, the non-linear system (8) can be written as linearization

$$
\mathbf{x}_{t+1} \approx J \mathbf{x}_{t},
$$

where $J$ is a Jacobi matrix determined at the fixed-point $\mathbf{x}_{F}$.

The next step is to examine the stability of the linear approximation and to draw conclusions concerning the non-linear system. According to the Lyapunov method, a non-linear system is locally stable asymptotically when its linear approximation is asymptotically stable at this point. A non-linear system is unstable if its linear approximation is also unstable. However, if the linear approximation is stable, but not necessarily asymptotically, nothing can be concluded about the stability of the primary non-linear system.

\section{Experiment}

In this section, qualitative and quantitative methods used in this paper to assess control algorithms are enumerated and described more precisely.

6.1. Qualitative assessment: Simulation diagrams. For each algorithm presented in the article, the following diagrams and charts are performed and analysed for given $\varepsilon$ and $\mathbf{p}$ :

- the diagram illustrating the lattice behavior in time and the space domains described by $x_{t}(n)=f(n, t)$,
- the chart illustrating the behavior of the selected component $n=25$ in time described by $x_{t}(25)=$ $f(t)$,

- the chart illustrating the behavior of all components in space in selected time step $t=150$ described by $x_{150}(n)=f(n)$

- the chart illustrating the distribution of Lyapunov exponents in space (for all components) described by $h=f(n)$

- the chart illustrating the distribution of the net direction phase indicators in space (for all components) described by $M=f(n)$.

The first three diagrams illustrate the trajectories generated by the system in time and space, while the charts contain the distribution of $h$ and $M$ and provide quantitative information about the type of behavior in the system.

6.2. Quantitative assessment: Indicators. The first indicator provides information about the deviation from the fixed point $x_{F}$ averaged over time and space. This indicator is calculated based on the following equation:

$$
q=\frac{1}{N T} \sum_{n=1}^{N} \sum_{t=1}^{T}\left|x_{t}(n)-x_{F}\right| .
$$

The next two indicators provide information about minimal and maximal values of Lyapunov exponents in space:

$$
\begin{aligned}
h_{\min } & =\min \left\{h_{1}, h_{2}, \ldots, h_{N}\right\}, \\
h_{\max } & =\max \left\{h_{1}, h_{2}, \ldots, h_{N}\right\},
\end{aligned}
$$

where $h_{1}, h_{2}, \ldots, h_{N}$ are values calculated based on $h_{\max }(n)$ from Eqns. (23). Apart from the above mentioned indicators, the average value $\left(h_{\text {avg }}\right)$ and the standard deviation $\left(s_{h}\right)$ are calculated for Lyapunov exponents distributed in space.

Similar indicators are proposed for the net direction phase:

$$
\begin{aligned}
& M_{\min }=\min \left\{M_{1}, M_{2}, \ldots, M_{N}\right\}, \\
& M_{\max }=\max \left\{M_{1}, M_{2}, \ldots, M_{N}\right\},
\end{aligned}
$$

where $M_{1}, M_{2}, \ldots, M_{N}$ are calculated based on Eqn. (24). The average value $\left(M_{\mathrm{avg}}\right)$ and standard deviation $\left(s_{M}\right)$ are also calculated for the net direction phase. The first indicator denoted by $q$ provides some basic information about the quality of control. The other defined indicators provide some view on the type of behavior generated by each component in space.

6.3. Control algorithms. Table 1 contains a definition of basic control algorithms examined in the paper. 


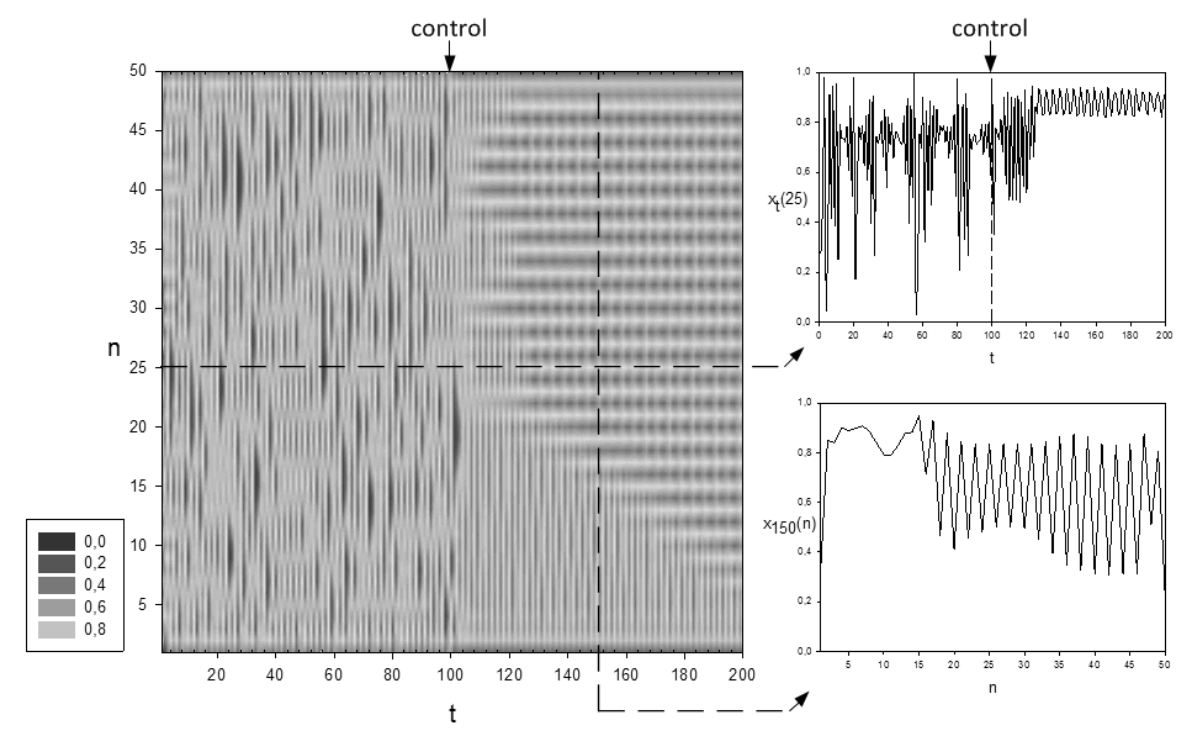

Fig. 1. Diagram illustrating lattice behavior for the system without and under control of the algorithm A1 $(K=0.32)$ in time and space domains for $\varepsilon=0.8$ and $p=4$.

Table 1. Control algorithms.

\begin{tabular}{|c|c|}
\hline Algorithm & Equation \\
\hline \hline $\mathrm{A} 1$ & $u_{t}(n)=K\left[x_{t}(n)-x_{F}\right]$ \\
$\mathrm{A} 2$ & $u_{t}(n)=K\left[x_{t}(n)-x_{t-1}(n)\right]$ \\
$\mathrm{A} 3$ & $u_{t}(n)=K\left[\sum_{j=-1}^{1}\left(x_{t}(n+j)-x_{F}\right)\right]$ \\
\hline
\end{tabular}

\section{Algorithm A1}

7.1. System equation. The following equation describes the system with the control algorithm A1:

$$
\begin{aligned}
x_{t+1}(n)= & (1-\varepsilon) g\left(x_{t}(n)\right) \\
& +(\varepsilon / 2)\left[g\left(x_{t}(n-1)\right)+g\left(x_{t}(n+1)\right)\right] \\
& +K\left[x_{t}(n)-x_{F}\right] .
\end{aligned}
$$

7.2. Stability analysis. In order to perform a stability analysis for the system described by Eqn. (33), there is a need to calculate partial derivatives:

$\frac{\partial x_{t+1}(n)}{\partial x_{t}(m)}= \begin{cases}(\varepsilon / 2) \Lambda=B & \text { for } m=n-1, \\ (1-\varepsilon) \Lambda+K=A & \text { for } m=n, \\ (\varepsilon / 2) \Lambda=B & \text { for } m=n+1, \\ 0 & \text { for }|m-n|>1,\end{cases}$

where

$$
\Lambda=\left.\frac{\partial g(x)}{\partial x}\right|_{x=x_{F}} .
$$

As was shown by Korus (2011), the eigenvalues of the system's Jacobi matrix can be calculated by using

$$
\begin{aligned}
& \lambda_{1}=A+2 B=\Lambda+K, \\
& \lambda_{2}=A-2 B=\Lambda(1-2 \varepsilon)+K .
\end{aligned}
$$

The system (33) is stable if $\left|\lambda_{i}\right|<1$ is fulfilled for each $i$, which also means

$$
\left\{\begin{array}{l}
-1<\lambda_{1}<1 \\
-1<\lambda_{2}<1
\end{array}\right.
$$

Since $\Lambda=-2$ for $p=4$ and $\varepsilon=0.8$, to fullfil the first of the above conditions, the gain of feedback loop should be selected from the range $K \in(1,3)$. The second condition introduces restrictions $K \in(-2.2,-0.2)$. These ranges are contradictory, which means that this system is not stable.

7.3. Numerical simulation. Figure 1 contains diagrams which illustrate CML behavior in time and space for parameters $\varepsilon=0.8, p=4$ and $K=0.32$. For time steps greater than $t=100$, external control is turned on. As can be seen in the diagrams, there is a change in the system's behavior after the time step $t=100$. Before this time step, the system generates strongly disordered behavior while for time steps $t>100$ the behavior of the system seems to be more ordered and some patterns can be identified. A change in the system's behavior can be observed on charts: $x_{t}(n)=f(n, t)$ and $x_{t}(25)=f(t)$. The third chart illustrates the distribution of cell values in space for the time step $t=150$, i.e., when external control is turned on. Based on these three diagrams, it can be only stated that there is a noticeable change in the system's behavior, but the trajectories of the system with external control are probably not periodic for all of the system's components.

Figure 2 illustrates the distribution of Lyapunov exponents for all components for the system without and 


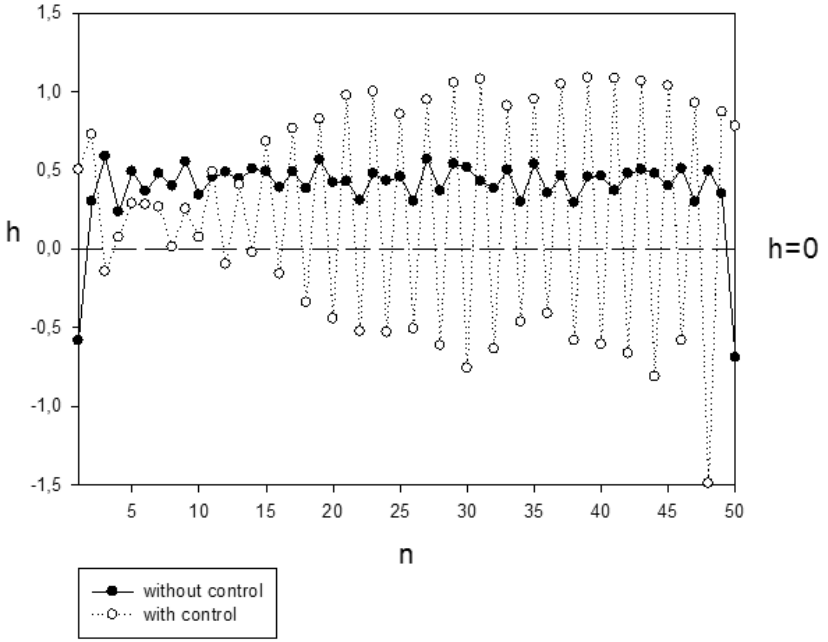

Fig. 2. Lyapunov exponents for all components $h(n)$ for the system without and under control of the algorithm A1 ( $K=0.32$ ) for $p=4$ and $\varepsilon=0.8$.

with control. As one can see, almost all values (apart from two edge ones, which are related to boundary conditions) are positive for the system without control. This means that the trajectories generated by the system are chaotic. When external control is turned on, some values become negative, which means that the trajectories in these cells are more ordered now.

Figure 3 presents the value distribution of the net direction phase indicator for all the components for the system without and with control. As can be observed, for some components in space, $M$ is closer to 0 . This means that the trajectories for these components switch symmetrically from the up direction phase to the down direction phase and the system behaves regularly. To summarize, this type of system control gives insufficient results. External control changes the system's behavior, but not to a periodic one and not for all of the system's components.

Table 2 contains values of indicators defined in one of the previous sections for the system without control and with control by using the algorithm A1. As can be noticed, $h_{\text {min }}$ is much lower when the control algorithm is turned on, while $h_{\max }$ is a bit higher. This change, together with the fact that $h_{\text {avg }}$ is decreased and $s_{h}$ is increased, means that this algorithm is able to change the behavior of the system, but only in a limited number of components. A similar conclusion can be made based on values of the net direction phase indicator. The change in the behavior is evidently noticeable in the system, but the trajectories of the system with external control are not periodical and not in all components.

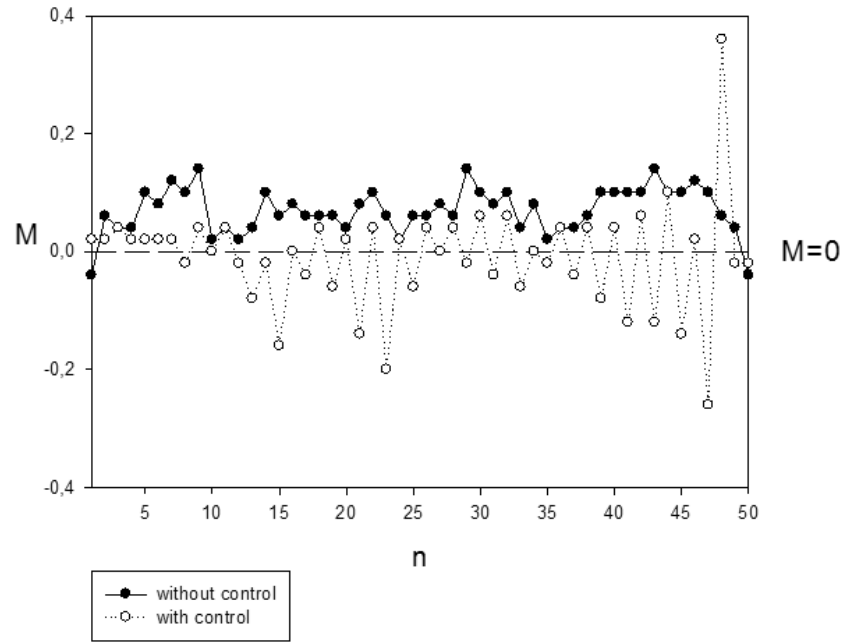

Fig. 3. Net direction phase for all components $M(n)$ for the system without and under control of the algorithm A1 ( $K=0.32$ ) for $p=4$ and $\varepsilon=0.8$.

Table 2. Values of all indicators for the system without and under control of the algorithm A1 $(K=0.32)$.

\begin{tabular}{|c|c|c|}
\hline Control & Indicator & Value \\
\hline \hline off & $h_{\min }$ & -0.69 \\
off & $h_{\max }$ & 0.59 \\
off & $h_{\text {avg }}$ & 0.39 \\
off & $s_{h}$ & 0.23 \\
off & $M_{\min }$ & -0.04 \\
off & $M_{\max }$ & 0.14 \\
off & $M_{\text {avg }}$ & 0.07 \\
off & $s_{M}$ & 0.04 \\
\hline on & $q$ & 0.34 \\
\hline on & $h_{\min }$ & -1.49 \\
on & $h_{\max }$ & 1.08 \\
on & $h_{\text {avg }}$ & 0.22 \\
on & $s_{h}$ & 0.69 \\
on & $M_{\min }$ & -0.26 \\
on & $M_{\max }$ & 0.36 \\
on & $M_{\text {avg }}$ & -0.01 \\
on & $s_{M}$ & 0.09 \\
\hline
\end{tabular}

\section{Algorithm A2}

8.1. System equation. Equation (37) defines a system similar to the previous one (33), but this time, instead of fixed point $x_{F}$, the value of the component in the previous time step $x_{t-1}(n)$ is used in the control element,

$$
\begin{aligned}
x_{t+1}(n)= & (1-\varepsilon) g\left(x_{t}(n)\right) \\
& +(\varepsilon / 2)\left[g\left(x_{t}(n-1)\right)+g\left(x_{t}(n+1)\right)\right] \\
& +K\left[x_{t}(n)-x_{t-1}(n)\right] .
\end{aligned}
$$




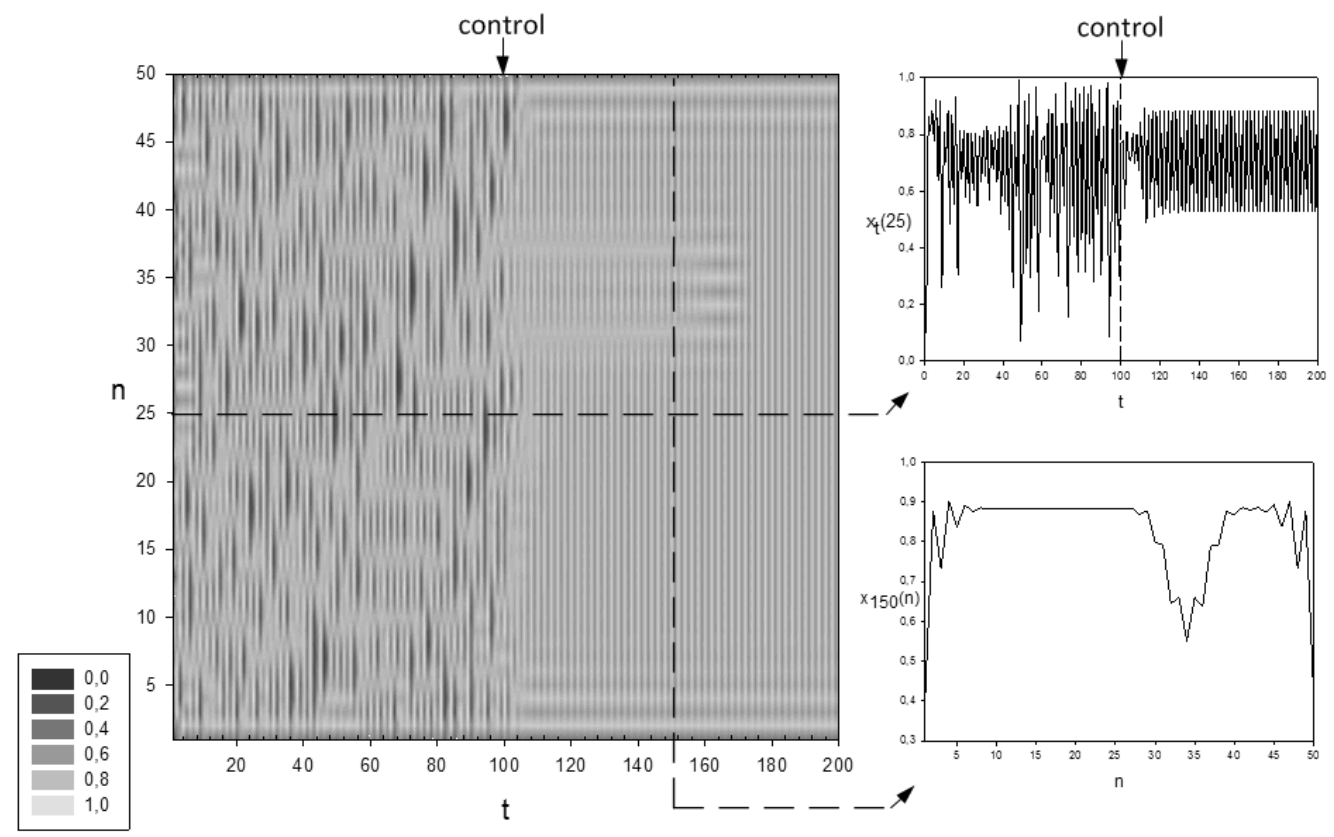

Fig. 4. Diagram illustrating the lattice behavior for the system without and under control of the algorithm A2 $(K=0.32)$ in time and space domains for $\varepsilon=0.8$ and $p=4$.

8.2. Stability analysis. Stability analysis of the system defined by Eqn. (37) can be made in much the same way as for the previous one. The nonlinear system defined by (37) can be approximated in a small neighborhood of the fixed point $x_{F}$ according to the equation (18):

$$
\delta \mathbf{x}_{t+1}=(J+B K) \delta \mathbf{x}_{t}
$$

where $\delta \mathbf{x}_{t+1}=\mathbf{x}_{t+1}-\mathbf{x}_{t}, \delta \mathbf{x}_{t}=\mathbf{x}_{t}-\mathbf{x}_{t-1}, J$ is a Jacobi matrix calculated for the fixed point $x_{F}, K$ is a gain matrix and $B=I$. To keep the system stable, the matrix $J^{\prime}=$ $(J+B K)$ must be stable. This gives exactly the same solution as for the control algorithm A1, which means that there is no $K$ which fulfills the stability conditions.

8.3. Numerical simulation. Figure 4 presents the lattice behavior for the system without and under control of the algorithm A2, both in time and space domains. Analysing all diagrams placed in the figure, it can be stated that the behavior of the system under control of the algorithm A2 is more ordered than previously, both in time and space. The chart $x_{t}(25)=f(t)$ shows a periodic-like state trajectory around fixed point $x_{F}=$ 0.75 . Moreover, the values of almost all the components in space seem to change synchronously apart from the area around $n>30$ and $n<40$, where some disturbances appear. This can be also observed in the chart $x_{150}(n)=$ $f(n)$.

Figure 5 illustrates the distribution of Lyapunov exponents for all the components for the system without

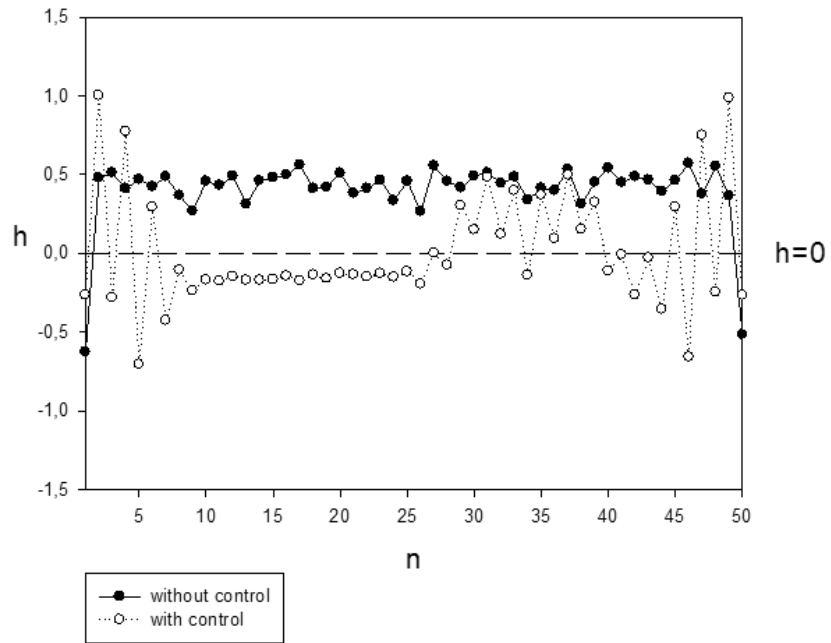

Fig. 5. Lyapunov exponents for all components $h(n)$ for the system without and under control of the algorithm A2 ( $K=0.32$ ) for $p=4$ and $\varepsilon=0.8$.

and under control. Based on this diagram, it can be stated that in the area $n \in(7,26)$ or $n \in(40,44)$ the system generates an ordered behavior for most of the space components. Lyapunov exponents are negative in these areas. This corresponds to the behavior which is visible in the diagrams in Fig. 5 .

Figure 6 confirms the change in the system's 


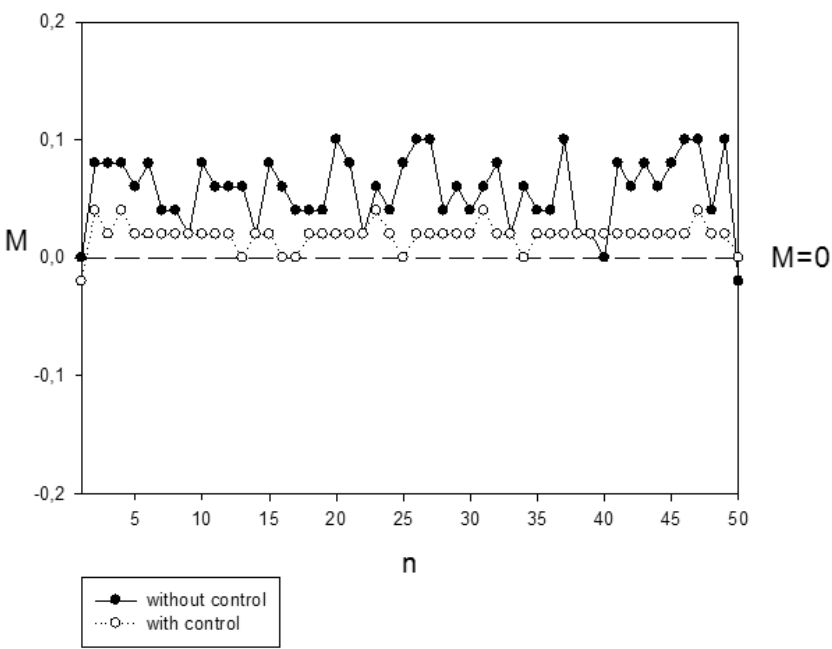

Fig. 6. Net direction phase for all components $M(n)$ for the system without and under control of the algorithm A2 ( $K=0.32$ ) for $p=4$ and $\varepsilon=0.8$.

behavior to a more ordered one when the control algorithm A2 is turned on. The values of the net direction phase indicator for all components of the system under control are closer to the value of $M=0$ than for the system without control, which confirms a more ordered behavior.

All values of indicators placed in Table 3 confirm conclusions which were drawn before. Based on those data, it can be stated that the system behavior changes to a more ordered one when the control algorithm A2 is turned on. The average deviation $q$ from the fixed point $x_{F}$ is lower for this algorithm than for the previous one, which means that control is more precise.

However, as the can be seen in all diagrams, the control algorithm changes behavior of the system from chaotic to a more ordered one or even periodic, but only for a limited set of components.

\section{Algorithm A3}

9.1. System equation. The following equation defines a system under control of the algorithm A3, which uses the values of surrounding components of $x_{t}(n)$ :

$$
\begin{aligned}
x_{t+1}(n)= & (1-\varepsilon) g\left(x_{t}(n)\right) \\
& +(\varepsilon / 2)\left[g\left(x_{t}(n-1)\right)+g\left(x_{t}(n+1)\right)\right] \\
& +K\left[\sum_{j=-1}^{1}\left(x_{t}(n+j)-x_{F}\right)\right] .
\end{aligned}
$$

Table 3. Values of all indicators for the system without and under control of the algorithm A2 $(K=0.32)$.

\begin{tabular}{|c|c|c|}
\hline Control & Indicator & Value \\
\hline \hline off & $h_{\min }$ & -0.63 \\
off & $h_{\max }$ & 0.57 \\
off & $h_{\text {avg }}$ & 0.40 \\
off & $s_{h}$ & 0.21 \\
off & $M_{\min }$ & -0.02 \\
off & $M_{\max }$ & 0.10 \\
off & $M_{\text {avg }}$ & 0.06 \\
off & $s_{M}$ & 0.03 \\
\hline on & $q$ & 0.16 \\
\hline on & $h_{\min }$ & -0.70 \\
on & $h_{\max }$ & 1.00 \\
on & $h_{\text {avg }}$ & 0.00 \\
on & $s_{h}$ & 0.36 \\
on & $M_{\min }$ & -0.02 \\
on & $M_{\max }$ & 0.04 \\
on & $M_{\text {avg }}$ & 0.02 \\
on & $s_{M}$ & 0.01 \\
\hline
\end{tabular}

9.2. Stability analysis. The stability analysis for the system defined by (39) can be made as follows:

$$
\frac{\partial x_{t+1}(n)}{\partial x_{t}(m)}= \begin{cases}(\varepsilon / 2) \Lambda+K=B & \text { for } m=n-1 \\ (1-\varepsilon) \Lambda+K=A & \text { for } m=n \\ (\varepsilon / 2) \Lambda+K=B & \text { for } m=n+1 \\ 0 & \text { for }|m-n|>1\end{cases}
$$

where

$$
\Lambda=\left.\frac{\partial g(x)}{\partial x}\right|_{x=x_{F}} .
$$

The eigenvalues of the system's Jacobi matrix can be calculated using

$$
\begin{aligned}
& \lambda_{1}=A+2 B=\Lambda+3 K, \\
& \lambda_{2}=A-2 B=\Lambda(1-2 \varepsilon)-K .
\end{aligned}
$$

The system (39) is stable if $\left|\lambda_{i}\right|<1$ for each $i$, which also means

$$
\left\{\begin{array}{l}
-1<\lambda_{1}<1 \\
-1<\lambda_{2}<1
\end{array}\right.
$$

The first condition introduces the restriction $K \in$ $(0.33,1)$ while the second one produces $K \in(0.2,2.2)$. Taking into consideration both the conditions, to keep the system stable, the gain of feedback loop should be selected from the range $K \in(0.33,1)$.

9.3. Numerical simulation. Figure 7 contains diagrams presenting the behavior of the system defined by Eqn. 39 without control and when the control is turned on. Analysing these diagrams, it can be stated that there is a significant change in the system's behavior. State trajectories change from chaotic to periodic or even approach a fixed point for almost all of the system's 


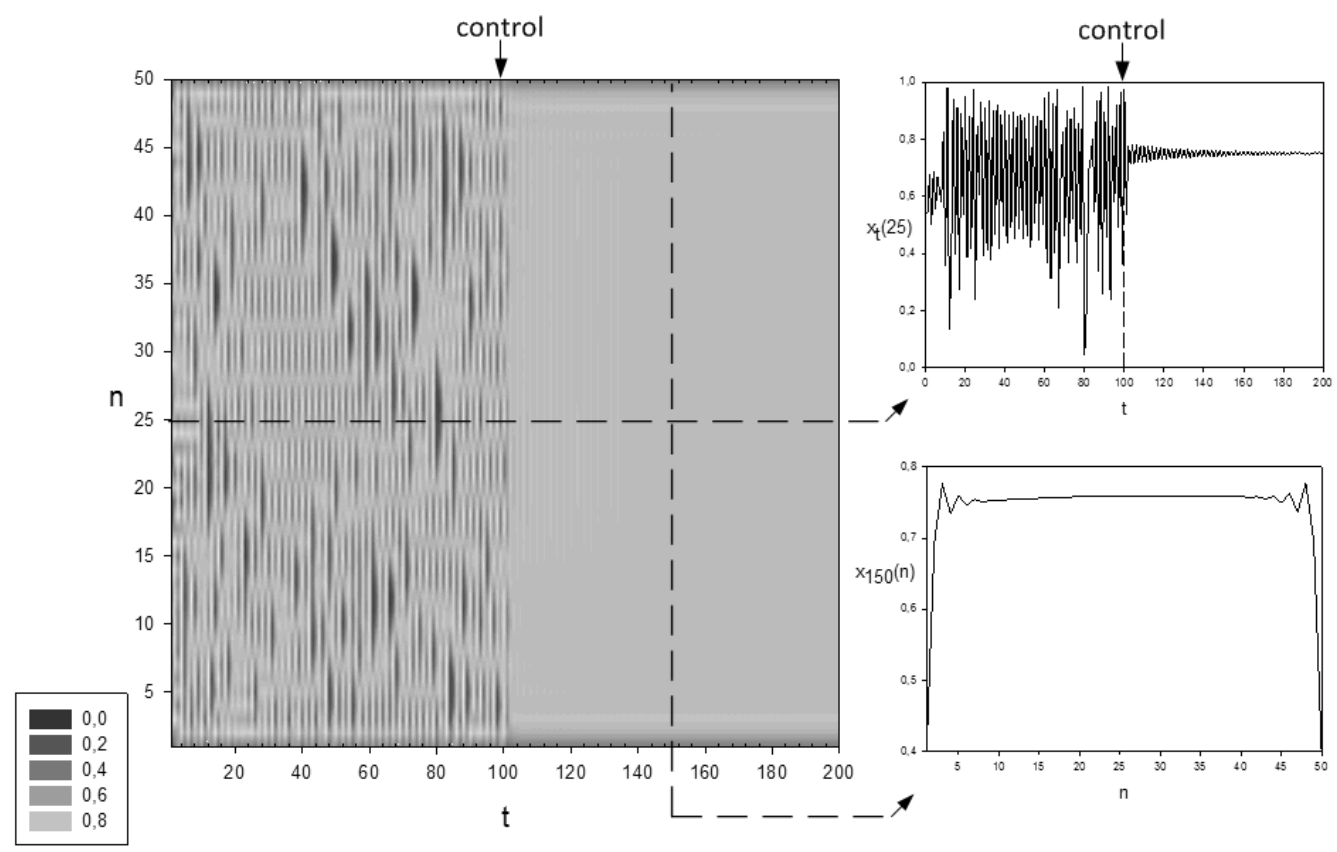

Fig. 7. Diagram illustrating the lattice behavior for the system without and under control of the algorithm A3 $(K=0.34)$ in time and space domains for $\varepsilon=0.8$ and $p=4$.

components. In particular, the state trajectory for the element $n=25$ oscillates around the fixed point $x_{F}=$ 0.75 , which can be observed in the diagram $x_{t}(25)=$ $f(t)$. Based on the diagram $x_{150}(n)=f(n)$, it can be stated, that the values of almost all components seem to change synchronously.

Figure 8 illustrates distribution of Lyapunov exponents for all of the system's components. As one can see, all values are strongly negative when the control is turned on. This means that the state trajectories for all components are ordered. Moreover, almost all Lyapunov exponents for the system without control are positive, which means that system without control is chaotic. Only the values of edge Lyapunov exponents are negative for the system without control, which is related to fixed boundary conditions.

Figure 9 presents the value distribution of the net direction phase indicator for all of the system's components for the system without and under control. This diagram also confirms that a significant change occurs in the system. As can be seen, when the control algorithm is turned on, almost all values are closer to zero. This means that almost all state trajectories are more ordered now.

All conclusions drawn above for the control algorithm A3 are also confirmed by the values of indicators collected in Table 4. First of all, an average value of deviation from the fixed point is about five or even ten times lower than for the previous algorithms. This confirms additionally the asymptotic stability of this

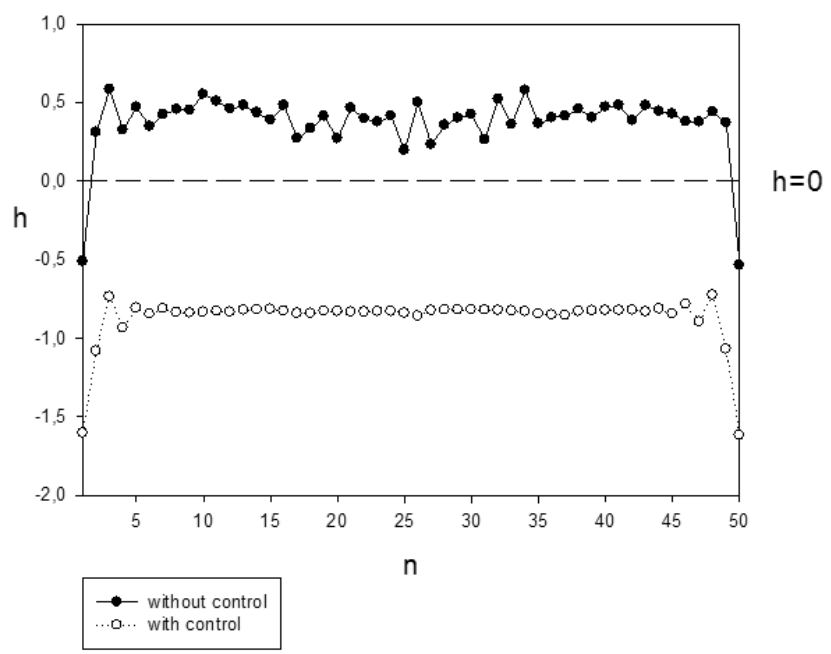

Fig. 8. Lyapunov exponents for all components $h(n)$ for the system without and under control of the algorithm A3 ( $K=0.34$ ) for $p=4$ and $\varepsilon=0.8$.

algorithm. Moreover, the average value of Lyapunov exponents is strongly negative, which, together with the fact that standard deviation for the system under control is lower, means that this algorithm efficiently changes the chaotic behavior to ordered one. 


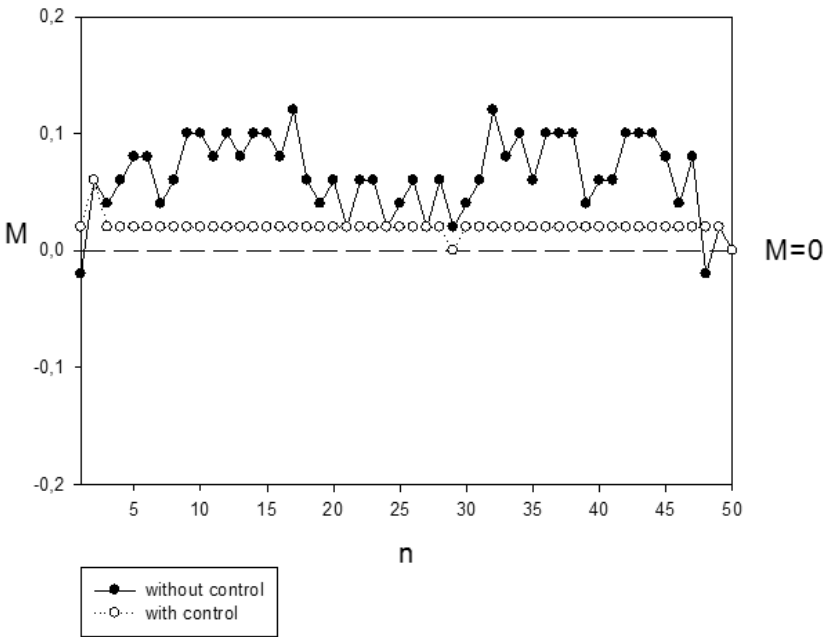

Fig. 9. Net direction phase for all components $M(n)$ for the system without and under control of the algorithm A3 ( $K=0.34$ ) for $p=4$ and $\varepsilon=0.8$.

Table 4. Values of all indicators for the system without and under control of the algorithm A3 $(K=0.34)$.

\begin{tabular}{|c|c|c|}
\hline Control & Indicator & Value \\
\hline \hline off & $h_{\min }$ & -0.54 \\
off & $h_{\max }$ & 0.58 \\
off & $h_{\text {avg }}$ & 0.37 \\
off & $s_{h}$ & 0.20 \\
off & $M_{\min }$ & -0.02 \\
off & $M_{\max }$ & 0.12 \\
off & $M_{\text {avg }}$ & 0.06 \\
off & $s_{M}$ & 0.03 \\
\hline on & $q$ & 0.03 \\
\hline on & $h_{\min }$ & -1.62 \\
on & $h_{\max }$ & -0.73 \\
on & $h_{\text {avg }}$ & -0.87 \\
on & $s_{h}$ & 0.16 \\
on & $M_{\min }$ & 0.00 \\
on & $M_{\max }$ & 0.06 \\
on & $M_{\text {avg }}$ & 0.02 \\
on & $s_{M}$ & 0.01 \\
\hline
\end{tabular}

\section{Conclusions}

The main aim of this paper was to present examination results of simple control algorithms for the purpose of controlling spatiotemporal chaos in the mathematical model named the coupled map lattice. As was mentioned, the task of the control of chaotic behaviors in spatially distributed systems is essential because such a type of system is common in many branches of science and industry. Moreover, as was shown in this paper, this task is not trivial if it is assumed that a change in behavior should be present for all components in the net.
To compare the quality of control in the meaning of changing the chaotic behavior to a periodic one, the entire framework presented in our previous paper (Korus, 2011) was used. In the first step of the control algorithm examination, the indirect Lyapunov method was used to estimate the values of the feedback gain $K$. Further, results of numerical simulation were provided. For each algorithm, diagrams illustrating the behavior of the system were included. Moreover, the values of the Lyapunov exponents and the net direction phase were calculated and presented for all of the system's components. Furthermore, bearing in mind the distributed nature of the CML system, the average values and the standard deviation for all components were included for both indicators.

Comparing the results of qualitative numerical simulation, i.e., the diagrams presenting the behaviors of the system without and under control, it can be noticed that state trajectories for the system under control of the algorithm A3 are more ordered for all of the system's components than for the algorithms A1 and A2. Furthermore, the algorithm A3 is the only one which makes the trajectories approach the fixed point, which is in line with the fact that the system is asymptotically stable. This conclusion was confirmed by quantitative results, i.e., the values of Lyapunov components, the net direction phase and statistics calculated for all systems components. Generally, the results obtained for the algorithm A3 are much better than for the other ones.

The fact that the third algorithm produced better results than other algorithms is connected with its construction, which takes into account the impact of surrounding components. This means that, while constructing a control method for spatially distributed systems with a regular structure, one should study the connection between surrounding components of the system first and implement them in the feedback module. Moreover, it is essential to assess the correlations in time and space. Only such a type of information increases the chance of building an efficient algorithm.

To gather information about the connection between components in the system, a linear method like autocorrelation can be used. However, since we are dealing with nonlinear systems, it is much better to use the mutual information indicator. Moreover, it should be taken into consideration that all results obtained in this article were obtained by examining the CML with fixed boundary conditions. This model is much easier to control than the CML with cyclic boundary conditions. It should also be noticed that the algorithm A3 is aggressive and changes the behavior of the system diametrically. It would be better to use it only in a small neighborhood of fixed point. 


\section{References}

Abarbanel, H. (1996). Analysis of Observed Chaotic Data, Springer-Verlag, New York, NY.

Alsing, P.M., Gavrielides, A. and Kovanis, V. (1994). Using neural networks for controlling chaos, Physical Review E 49(2): 1225-1231.

Andrievskii, B.R. and Fradkov, A.L. (2003). Control of chaos: Methods and applications, Automation and Remote Control 64(5): 673-713.

Andrzejak, R.G., Lehnertz, K., Mormann, F., Rieke, C., David, P. and Elger, C.E. (2001). Indications of nonlinear deterministic and finite-dimensional structures in time series of brain electrical activity: Dependence on recording region and brain state, Physical Review E 64(1): 1-8.

Argoul, F., Arneodo, A., Richetti, P. and Roux, J.C. (1987). From quasiperiodicity to chaos in the Belousov-Zhabotinskii reaction, I: Experiment, Journal of Chemical Physics 86(6): 3325-3339.

Banerjee, S., Misra, A.P., Shukla, P.K. and Rondoni, L. (2010). Spatiotemporal chaos and the dynamics of coupled Langmuir and ion-acoustic waves in plasmas, Physical Review E 81(1): 1-10.

Bashkirtseva, I. and Ryashko, L. (2013). Attainability analysis in the problem of stochastic equilibria synthesis for nonlinear discrete systems, International Journal of Applied Mathematics and Computer Science 23(1): 5-16, DOI: 10.2478/amcs-2013-0001.

Boukabou, A. and Mansouri, N. (2005). Predictive control of higher dimensional chaos, Nonlinear Phenomena in Complex Systems 8(3): 258-265.

Chen, G. and Dong, X. (1993). On feedback control of chaotic continuous-time systems, IEEE Transactions on Circuits and Systems 40(9): 591-601.

Chen, Q., Teng, Z. and Hu, Z. (2013). Bifurcation and control for a discrete-time prey-predator model with Holling-IV functional response, International Journal of Applied Mathematics and Computer Science 23(2): 247-261, DOI: 10.2478/amcs-2013-0019.

Chui, S.T. and Ma, K.B. (1982). Nature of some chaotic states for Duffing's equation, Physical Review A 26(4): 2262-2265.

Cordoba, A., Lemos, M.C. and Jimenez-Morales, F. (2006). Periodical forcing for the control of chaos in a chemical reaction, Journal of Chemical Physics 124(1): 1-6.

Crutchfield, J.P. and Kaneko, K. (1987). Directions in Chaos: Phenomenology of Spatio-Temporal Chaos, World Scientific Publishing Co., Singapore.

Dressler, U. and Nitsche, G. (1992). Controlling chaos using time delay coordinates, Physical Review Letters 68(1): $1-4$.

Gautama, T., Mandic, D.P. and Hulle, M.M.V. (2003). Indications of nonlinear structures in brain electrical activity, Physical Review E 67(1): 1-5.
Govindan, R.B., Narayanan, K. and Gopinathan, M.S. (1998). On the evidence of deterministic chaos in ECG: Surrogate and predictability analysis, Chaos 8(2): 495-502.

Gunaratne, G.H., Lisnay, P.S. and Vinson, M.J. (1989). Chaos beyond onset: A comparison of theory and experiment, Physical Review Letters 63(1): 1-4.

Held, G.A., Jeffries, C. and Haller, E.E. (1984). Observation of chaotic behavior in an electron-hole plasma in GE, Physical Review Letters 52(12): 1037-1040.

Kaneko, K. (1990). Simulating Physics with Coupled Map Lattices, World Scientific Publishing Co., Singapore.

Korus, Ł. (2011). Simple environment for developing methods of controlling chaos in spatially distributed systems, International Journal of Applied Mathematics and Computer Science 21(1): 149-159, DOI: 10.2478/v10006-011-0011-4.

Langenberg, J., Pfister, G. and Abshagen, J. (2004). Chaos from Hopf bifurcation in a fluid flow experiment, Physical Review E 70(1): 1-5.

Lasota, A. and Mackey, M. (1997). Chaos, Fractals, and Noise, Springer, New York, NY.

Mitkowski, P.J. and Mitkowski, W. (2012). Ergodic theory approach to chaos: Remarks and computational aspects, International Journal of Applied Mathematics and Computer Science 22(2): 259-267, DOI: 10.2478/v10006-012-0019-4.

Ogorzałek, M.J., Galias, Z., Dabrowski, W. and Dabrowski, A. (1996a). Spatio-temporal co-operative phenomena in CNN arrays composed of chaotic circuits simulation experiments, International Journal of Circuit Theory and Applications 24(3): 261-268.

Ogorzałek, M.J., Galias, Z., Dabrowski, W. and Dabrowski, A. (1996b). Wave propagation, pattern formation and memory effects in large arrays of interconnected chaotic circuits, International Journal of Bifurcation and Chaos 6(10): 1859-1871.

Ott, E. (2002). Chaos in Dynamical Systems, Cambridge University Press, Cambridge.

Ott, E., Grebogi, C. and Yorke, J.A. (1990). Controlling chaos, Physical Review Letters 64(11): 1196-1199.

Parmananda, P. (1997). Controlling turbulence in coupled map lattice systems using feedback techniques, Physical Review E 56(1): 239-244.

Procaccia, I. and Meron, E. (1986). Low-dimensional chaos in surface waves: Theoretical analysis of an experiment, Physical Review A 34(4): 3221-3237.

Pyragas, K. (2001). Control of chaos via an unstable delayed feedback controller, Physical Review Letters 86(11): 2265-2268.

Sauer, T., Yorke, J., and Casdagli, M. (1991). Embedology, Journal of Statistical Physics 65(3-4): 579-616.

Singer, J., Wang, Y. and Haim, H.B. (1991). Controlling a chaotic system, Physical Review Letters 66(9): 1123-1125. 
Stark, J. (1999). Delay embeddings for forced systems, I: Deterministic forcing, Jouranl of Nonlinear Science 9(3): 255-332.

Takens, F. (1981). Detecting Strange Attractors in Turbulence, Springer-Verlag, Berlin.

Used, J. and Martin, J.C. (2010). Multiple topological structures of chaotic attractors ruling the emission of a driven laser, Physical Review E 82(1): 1-7.

Wei, W., Zonghua, L. and Bambi, H. (2000). Phase order in chaotic maps and in coupled map lattices, Physical Review Letters 84(12): 2610-2613.

Yamada, T. and Graham, R. (1980). Chaos in a laser system under a modulated external field, Physical Review Letters 45(16): 1322-1324.

Yim, G., Ryu, J., Park, Y., Rim, S., Lee, S., Kye, W. and Kim, C. (2004). Chaotic behaviors of operational amplifiers, Physical Review E 69(1): 1-4.

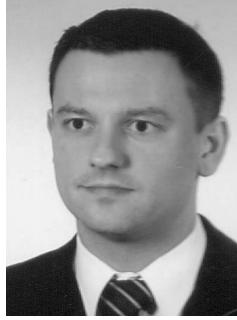

Lukasz Korus was born in 1978 in Poland. He received the M.Sc. degrees in automatic control, robotics and computer engineering from the Wrocław University of Technology, Poland, in 2003 and 2006, respectively. In 2009, he received a Ph.D. degree in the field of automatic control and robotics from the Wrocław University of Technology. Since 2009 he has been an assistant professor in the Institute of Computer Engineering, Control and Robotics at the same university. His research interests are chaos and control theories and their application to control of the chaotic behavior in spatially distributed systems.

Received: 7 July 2013 